\title{
An adaptive method for the numerical solution of a 2D Monge-Ampère equation
}

\author{
Alexandre Caboussat*, Dimitrios Gourzoulidis ${ }^{\dagger *}$ and Marco Picasso ${ }^{\dagger}$ \\ * Geneva School of Business Administration \\ University of Applied Sciences and Arts Western Switzerland \\ $\mathrm{CH}$ - 1227 Carouge, Switzerland \\ e-mail: alexandre.caboussat@hesge.ch,dimitrios.gourzoulidis@hesge.ch, \\ http://campus.hesge.ch/caboussat \\ ${ }^{\dagger}$ Institute of Mathematics \\ Ecole Polytechnique Fédérale de Lausanne \\ CH 1015- Lausanne, Switzerland \\ Email: marco.picasso@epfl.ch,dimitrios.gourzoulidis@epfl.ch
}

\begin{abstract}
Parabolic fully nonlinear equations may be found in various applications, for instance in optimal portfolio management strategy. We focus here on a canonical parabolic Monge-Ampère equation in two space dimensions. A numerical method has been investigated in [1]. The goal is to extend the methodology by coupling a time stepping semi-implicit method that relies on a conservative formulation of the Monge-Ampère equation with mesh adaptation.

The parabolic Monge-Ampère equation can be expressed as a strongly nonlinear, heat-type, parabolic equation, where the nonlinear diffusion function is expressed as a function of the cofactor matrix of the Hessian matrix of the solution. We linearize this diffusion operator and advocate a semi-implicit time-stepping algorithm. In particular, we use the time-evolutive equation to reach a stationary solution corresponding to a solution of the elliptic Monge-Ampère equation.
\end{abstract}

A low order, piecewise linear, finite element method is used for space discretization, together with a mixed approach for the approximation of the second derivatives. The error is bounded above by an error indicator plus an extra term that can be disregarded in special cases. A mesh adaptivity strategy based on these estimates is then implemented within the time-stepping algorithm for the nonlinear equation.

Numerical experiments exhibit appropriate convergence orders and a robust behavior. Adaptive mesh refinement proves to be efficient and accurate to tackle test cases with singularities. In particular, we consider equations with exact solutions with singularities on the boundary of the domain, or with righthand sides involving Dirac functions.

\section{REFERENCES}

[1] A. Caboussat, D. Gourzoulidis, "A second order time integration method for the approximation of a parabolic 2D Monge-Ampère equation", Proceedings of European Numerical Mathematics and Advanced Applications Conference 2019, F. Vermoelen, C. Vuik Eds, Lecture Notes in CSE, Springer (2020).

[2] R. Verfürth. A posteriori error estimates for nonlinear problems, finite element discretizations of elliptic equations. Math. Comput., 62(206): 445-475 (1994). 